\title{
CARACTERIZAÇÃO DO POTENCIAL DE BIODISPONIBILIDADE DE METAIS (Zn, Cd, Pb, Cu E Ni) EM SEDIMENTOS DE CORRENTE DO RIO SÃO FRANCISCO
}

\author{
Vasconcelos F. $\mathrm{M}^{1}$; Almeida, D. $\mathrm{F}^{2}$; Santos, L. ${ }^{3}$; Moreira, T. T. ${ }^{3}$ \\ RESUMO
}

\begin{abstract}
Em países com intensa atividade industrial, a contaminação de sedimentos por metais é relativamente comum e tem sido alvo de diversas pesquisas com o objetivo de determinar o impacto ambiental desse tipo de contaminação. Sabe-se hoje que somente a concentração total de metais nos sedimentos não é um bom parâmetro para a avaliação da toxicidade de metais em sedimentos. Além de ensaios de eco toxicidade é recomendável a realização de ensaio de caracterização geoquímica da concentração de sulfetos voláteis por acidificação e de metais simultaneamente extraídos (AVS/SEM) que é utilizada para a determinação da fração potencialmente biodisponível de metais em sedimento. Sete amostras em duplicada foram coletadas nas margens do rio São Francisco próximo a uma planta metalúrgica de zinco no município de Três Marias MG. Os ensaios de caracterização geoquímica do tipo AVS/SEM dessas amostras sugerem que apesar da concentração de metais totais ( $\mathrm{Zn}, \mathrm{Cd}, \mathrm{Pb}, \mathrm{Cu}$ e Ni) estarem acima do Nível 1 da norma ambiental de qualidade de sedimentos, o potencial de biodisponibilidade de metais nessas amostras é relativamente baixo para seis dentre sete amostras.
\end{abstract}

Palavras chaves: metais; contaminação; biodisponibilidade; sedimentos.

\begin{abstract}
In countries with intense industrial activities, sediment contamination by metals is relatively common and it has being the target of several researches with the goal of investigating the environmental impact assessment of this type of contamination. It is well known that using only the bulk metal concentration in sediment is not sufficient for estimating the metal toxicity in this media. Besides ecotoxicological tests it is recommended the determination acid volatile sulfides and simultaneously extracted metals (AVS/SEM) for the estimation of the fraction of potential bioavailability of metal in the sediment. Seven samples in duplicate were collected from the edges of the São Francisco River near a zinc metallurgical plant in the town of Três Marias MG. The sediment AVS/SEM geochemical characterization test results of these samples suggest that although the metal bulk concentrations $(\mathrm{Zn}, \mathrm{Cd}, \mathrm{Pb}, \mathrm{Cu}$ e $\mathrm{Ni}$ ) from these samples are above the Level 1 of the environmental regulation for sediment quality, six out of seven samples presented low potential for metal bioavailability.
\end{abstract}

Keywords: metals; contamination; bioavailability; sediments.

\section{INTRODUÇÃO}

Poluentes tóxicos presentes em sedimentos de lagos, rios e reservatórios criam uma situação potencial para a degradação da coluna de água localizada acima destes sedimentos, mesmo quando a qualidade de água é considerada como boa ou aceitável (U.S.EPA, 2005). Dessa forma, passa a ser imprescindível a compreensão da estabilidade química desse tipo de contaminante, em termos das condições físico-química que esse se encontra nos sedimentos, assim como, a caracterização das fases minerais que os controlam nesse meio. Devido ao grande número de fontes pontuais e não pontuais de metais tais como: Zinco (Zn), Cádmio (Cd), Chumbo $(\mathrm{Pb})$, Cobre $(\mathrm{Cu})$ e Níquel $(\mathrm{Ni})$ em uma metalúrgica e a sua natureza não degradável no meio ambiente esses metais assim como alguns contaminantes orgânicos não iônicos são compostos que podem ser classificados como de preocupação ambiental em termos de impacto a flora e a fauna local.

O método SVA/MES permite avaliar o potencial de biodisponibilidade de metais em sedimentos e o risco a organismos bentônicos através de ensaios rápidos e expeditos. Esse método, além de possibilitar uma predição simples e barata da toxicidade de um sedimento, é recomendado pela U.S.EPA e pela Environmental Directory of the European Commission, como uma forma de aquilatar a biodisponibilidade de metais presentes em sedimentos.

Em camadas oxidadas do sedimento, as fases minerais oxi-hidróxidos de ferro e de manganês geralmente controlam, principalmente, a biodisponibilidade desses metais-traço. Contudo, quando em camadas anóxicas, os parâmetros reguladores mais importantes são $\mathrm{pH}$ e sulfeto secundário. Essas condições anóxicas se desenvolvem em diversas profundidades, quando em presença de matéria orgânica ou de outros compostos, mesmo que a superfície do sedimento seja bem oxidada. Os sulfetos secundários, ou seja, precipitados em ambiente anóxico são gerados, principalmente, por bactérias sulfato-redutoras, quando em presença de matéria orgânica e condições físico-químicas para tal (ambiente redutor e pH próximo a 7,0). Esse tipo de sulfeto é principalmente, mono sulfetos amorfos de ferro $(\mathrm{FeS})$ ou sulfetos também amorfos de outros metais. 


\section{METODOLOGIA}

Fundamentação teórica

Alguns cátions metálicos divalentes $(\mathrm{Zn}, \mathrm{Cd}, \mathrm{Pb}, \mathrm{Cu}$ e Ni) por apresentarem uma constante de solubilidade $\left(\mathrm{K}_{\mathrm{sp}}\right)$ pequena, a exemplo do Cd com $\log \mathrm{K}_{\mathrm{sp}}=-14,36 \mathrm{e}$ do $\mathrm{Cu} \operatorname{com} \log \mathrm{K}_{\mathrm{sp}}=-34,65$ (Stumm e Morgan, 1996), são removidos da fração solúvel por precipitação dos sulfetos metálicos secundários (U.S. EPA, 2000), de acordo com a reação:

$$
\mathrm{Me}^{2+}{ }_{(\mathrm{aq})}+\mathrm{S}_{(\text {(aq) }}^{2-} \rightarrow \mathrm{MeS}_{(\mathrm{s})}
$$

A concentração de sulfetos secundários no sedimento pode ser dimensionada pelo tratamento da amostra com $\mathrm{HCl}$ a frio na concentração de $1 \mathrm{~mol} / \mathrm{L}$ (Di Toro et al., 1990;), segundo a reação a seguir:

$$
4 \mathrm{HCl}_{(\mathrm{aq})}+2 \mathrm{MeS}_{(\mathrm{s})} \rightarrow 2 \mathrm{Me}^{2+}{ }_{(\mathrm{aq})}+4 \mathrm{Cl}_{(\mathrm{aq})}^{-}+2 \mathrm{H}_{2} \mathrm{~S}_{(\mathrm{g})}
$$

O ácido clorídrico volatilizará o sulfeto que será transportado pelo nitrogênio $\left(\mathrm{N}_{2}\right)$ e será coletado em um recipiente apropriado onde é determinada a sua concentração através da sua reação com o composto de azul de metilamina. O sulfeto determinado dessa forma recebe o nome de SVA ("Sulfeto Volatilizado por Acidificação"). Já a fração dissolvida dos metais divalentes $(\mathrm{Zn}, \mathrm{Cd}, \mathrm{Pb}, \mathrm{Cu}$ e $\mathrm{Ni})$ extraídos pelo ácido clorídrico, representados nas equações acima (1 e 2) como $\mathrm{Me}^{2+}{ }_{(\mathrm{aq})}$, será analisada de forma que a soma molar desses metais representará a fração dos metais simultaneamente extraídos (MSE).

Muitos estudos têm demonstrado (Di Toro et al., 1990 Ankley et al., 1991) que a toxicidade, associada à concentração intersticial de metais, aos organismos bentônicos que vivem dentro dos sedimentos tipicamente não é manifestada quando a concentração de SVA no sedimento excede a soma molar da concentração de metais simultaneamente extraídos (MSE). Dessa forma, um sedimento com concentrações de metal total acima da concentração máxima permitida em termos de metais (CONAMA, 344/04) potencialmente não apresentaria risco se:

Em termos de concentração molar:

$\Sigma[\mathrm{MSE}]-(\mathrm{SVA}) \leq 0$; onde:

$\Sigma[\mathrm{MSE}]=$ Somatória das concentrações molares de $\mathrm{Zn}, \mathrm{Cd}, \mathrm{Pb}, \mathrm{Cu}$ e Ni

$\mathrm{SVA}=$ Concentração de sulfeto extraído por $\mathrm{HCl}$ $1 \mathrm{M}$.

\section{COLETA DE AMOSTRAS DE SEDIMENTO}

As amostras de sedimento para esse estudo foram coletadas em seis pontos com o auxílio de uma draga de aço inox tipo Petersen de $20 \mathrm{~kg}, 10 \mathrm{~L}$ de capacidade e área de coleta de $928 \mathrm{~cm}^{2}$, conforme apresenta a figura abaixo (Figura 1). As amostras foram identificadas e alojadas em potes de polipropileno com capacidade para $1 \mathrm{~kg}$ com tampas possuindo lacre e mantidas refrigeradas em caixas térmicas contendo gelo.

\section{INSTRUMENTAL}

A metodologia empregada para análise seguiu os procedimentos de “AVS-SEM" (U.S. EPA 821-R-91-

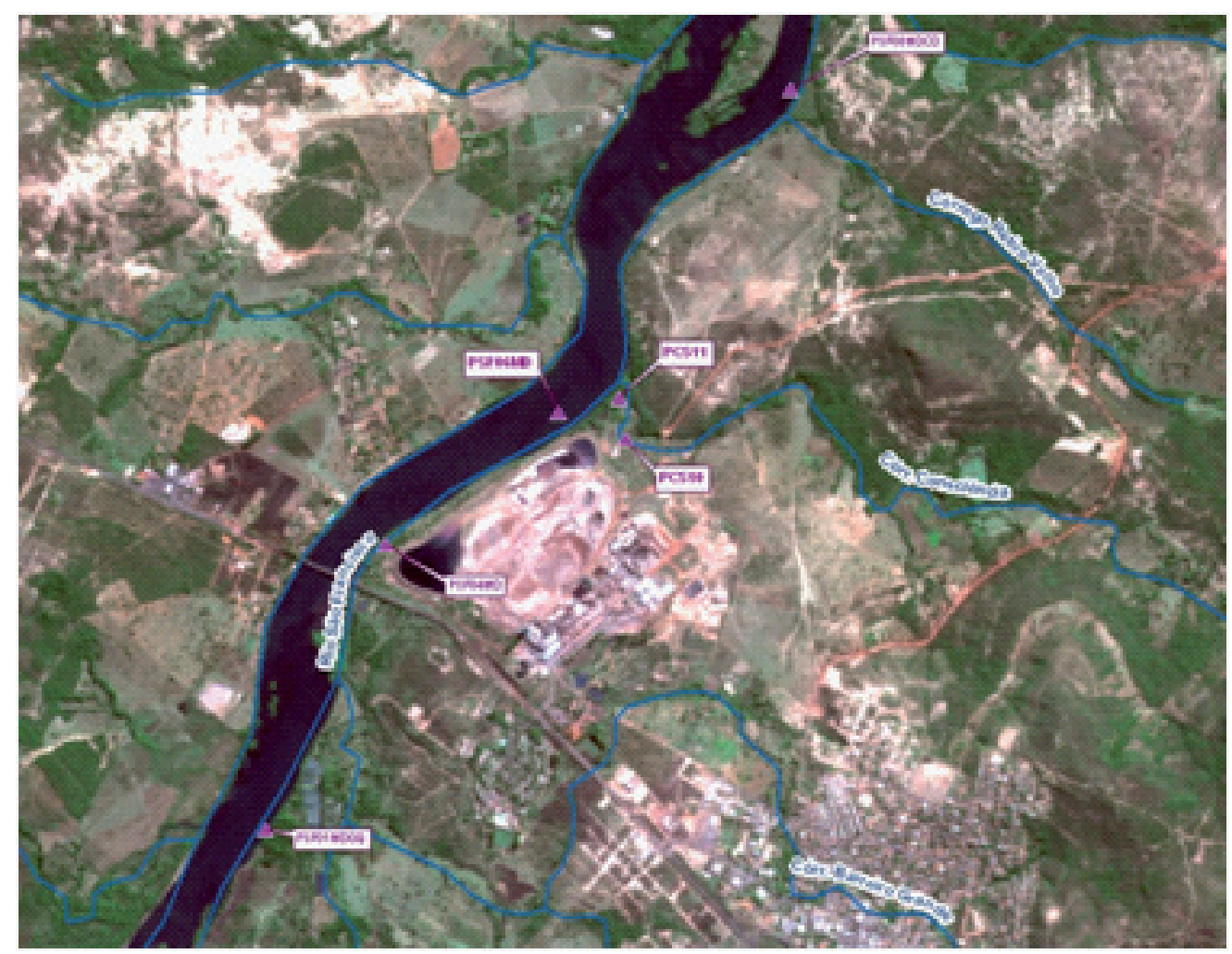

Figura 1 - Pontos de coleta de amostras de sedimento ao longo do Rio São Francisco para os testes de SVA-MSE 
100). A descrição do método de análise é apresentada a seguir.

A amostra de sedimento total (úmido) é pesada e introduzida no balão de reação juntamente com 100 mL de água Milli-Q. Fecha-se o balão e a suspensão é agitada durante fluxo de nitrogênio ultrapuro. Em seguida, introduzem-se $20 \mathrm{~mL}$ de $\mathrm{HCl} 6 \mathrm{M}$, previamente aerado com nitrogênio $\left(\mathrm{N}_{2}\right)$. O sulfeto secundário volatilizado é então arrastado pelo gás de arraste $\left(\mathrm{N}_{2}\right)$, através dos tubos de vidro que se encontram mergulhados dentro dos frascos receptores, por um tempo de 30 minutos. A suspensão restante do balão (MSE) é filtrada em membrana de $0,45 \mu \mathrm{m}$ para a determinação de metais ( $\mathrm{Zn}, \mathrm{Cd}, \mathrm{Pb}, \mathrm{Cu}$ e Ni). Para a determinação da fração SVA e MES faz-se uso dos seguintes métodos: SMEWW (2005) 3030 B, C, D, E, F, I, 3120 B.; 4500S2-D, F do STANDARD METHODS (2005).

Em resumo, o arranjo experimental (Figura 2) desse método consiste de um balão de reação onde o sedimento é atacado por uma solução ácida, seguido por dois tubos receptores conectados seqüencialmente para capturar o sulfeto volátil extraído pelo ataque ácido (SVA).

\section{RESULTADOS}

Conforme citado no item 2.1 deste relatório, efetuou-se a análise de AVS/SEM para as amostras

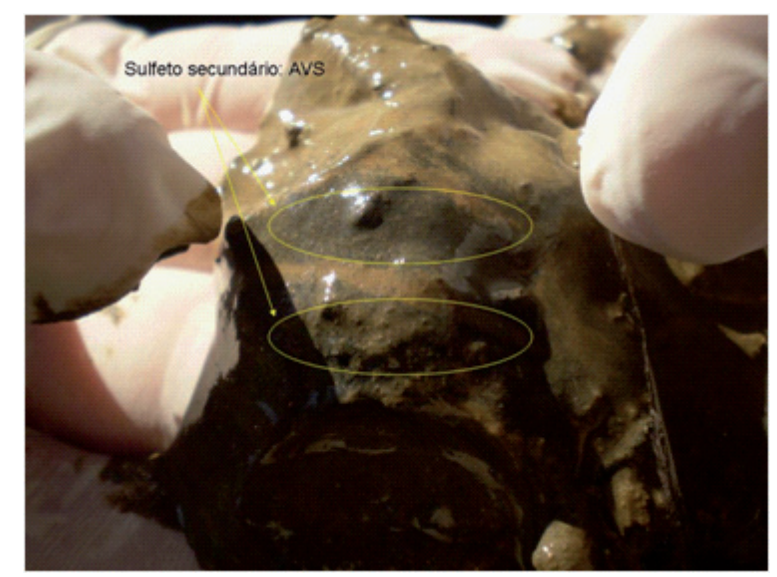

Figura 3 - Foto de sedimento do rio São Francisco com a presença de sulfetos secundários (manchas escuras dentro de um material silto argiloso marrom claro).

do porte do rio São Francisco, obtidos a jusante de uma planta metalúrgica de chumbo, através de estudos realizados por Chapman (2001). Conforme observado no oficio encaminhado pela Agência Nacional das Águas (ANA), no relatório da campanha passada de fato foi observada uma inconsistência da unidade reportada no relatório passado, o que poderia ter levado a interpretações errôneas, entretanto após averiguação da inconsistência pode ser observado que se tratava de um erro na editoração do documento (troca da unidade
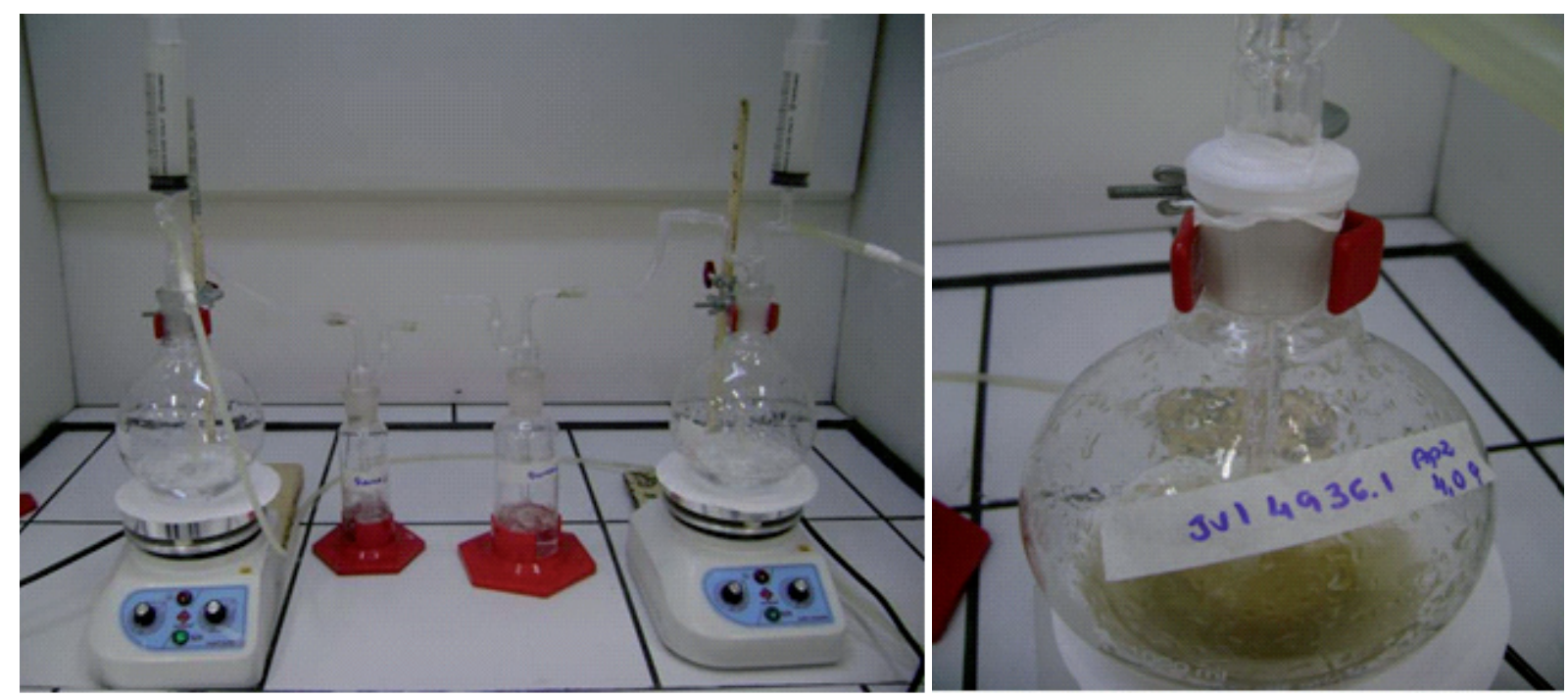

Figura 2 - Aparato experimental do método SVA-MSE.

consideradas como hot-spots e os valores encontrados são apresentados na Tabela 1 abaixo. Os resultados apresentados aqui passaram por uma avaliação de controle de qualidade que incluiu análise de branco e duplicatas e representam valores definitivos. Como referência, para comparação desses dados, utilizou-se os valores de AVS/SEM obtidos por Silvério (2003) em estudos realizados na represa Rasgão, localizada no Estado de São Paulo e dados do rio Missouri (EUA), de $\mu$ mol. g-1 por $\mu$ M. kg-1).

\section{CONCLUSÕES}

Os resultados apresentados aqui passaram por um rígido controle de qualidade que incluiu a análise de duplicatas e replicatas, durante o trabalho foi necessário inclusive a revisão do método analítico do laboratório responsável pela analise das amostras. Foi utilizado 
Tabela 1 - Valores dos ensaios de SVA/MSE das amostras do rio São Franscisco.

\begin{tabular}{|l|c|c|c|c|c|c|c|c|}
\hline $\begin{array}{c}\text { Ponto de } \\
\text { coleta }\end{array}$ & $\mathbf{C d}$ & $\mathbf{C u}$ & $\mathbf{N i}$ & $\mathbf{P b}$ & $\mathbf{Z n}$ & $\mathbf{S}^{2-}$ & (fcop) & $\begin{array}{c}\text { (MSE - } \\
\text { SVA)/fco }\end{array}$ \\
\hline \multicolumn{7}{|c|}{$\mu \mathrm{mol} / \mathbf{g}$} \\
\hline PSF01 & $<0,001$ & 0,192 & 0,042 & 0,065 & 0,266 & 0,002 & 0,008 & $\mathbf{7 3}$ \\
\hline PSF04 & 0,039 & 0,376 & 1,500 & 0,101 & 14,131 & 0,006 & 0,012 & $\mathbf{1 . 1 7 7}$ \\
\hline $\begin{array}{l}\text { PSF04 } \\
\text { (replicata) }\end{array}$ & 0,040 & 0,285 & 1,500 & 0,086 & 14,222 & 0,001 & 0,013 & $\mathbf{1 . 1 6 6}$ \\
\hline PSF06 & 0,266 & 0,574 & 0,099 & 0,499 & 25,172 & 0,007 & 0,011 & $\mathbf{2 . 3 1 7}$ \\
\hline PSF08 & $<0,001$ & 0,173 & $<0,003$ & 0,094 & 4,618 & 0,005 & 0,012 & $\mathbf{4 0 3}$ \\
\hline $\begin{array}{l}\text { Represa } \\
\text { Rasgão* }\end{array}$ & 0,02 & 0,87 & 0,65 & 0,37 & 9,6 & 36,0 & - & $\mathbf{- 2 4 , 7 0}$ \\
\hline $\begin{array}{l}\text { Rio } \\
\text { Missouri** }\end{array}$ & - & 0,06 & 0,05 & 0,13 & 0,11 & 8,4 & - & $\mathbf{- 8 , 1 0}$ \\
\hline
\end{tabular}

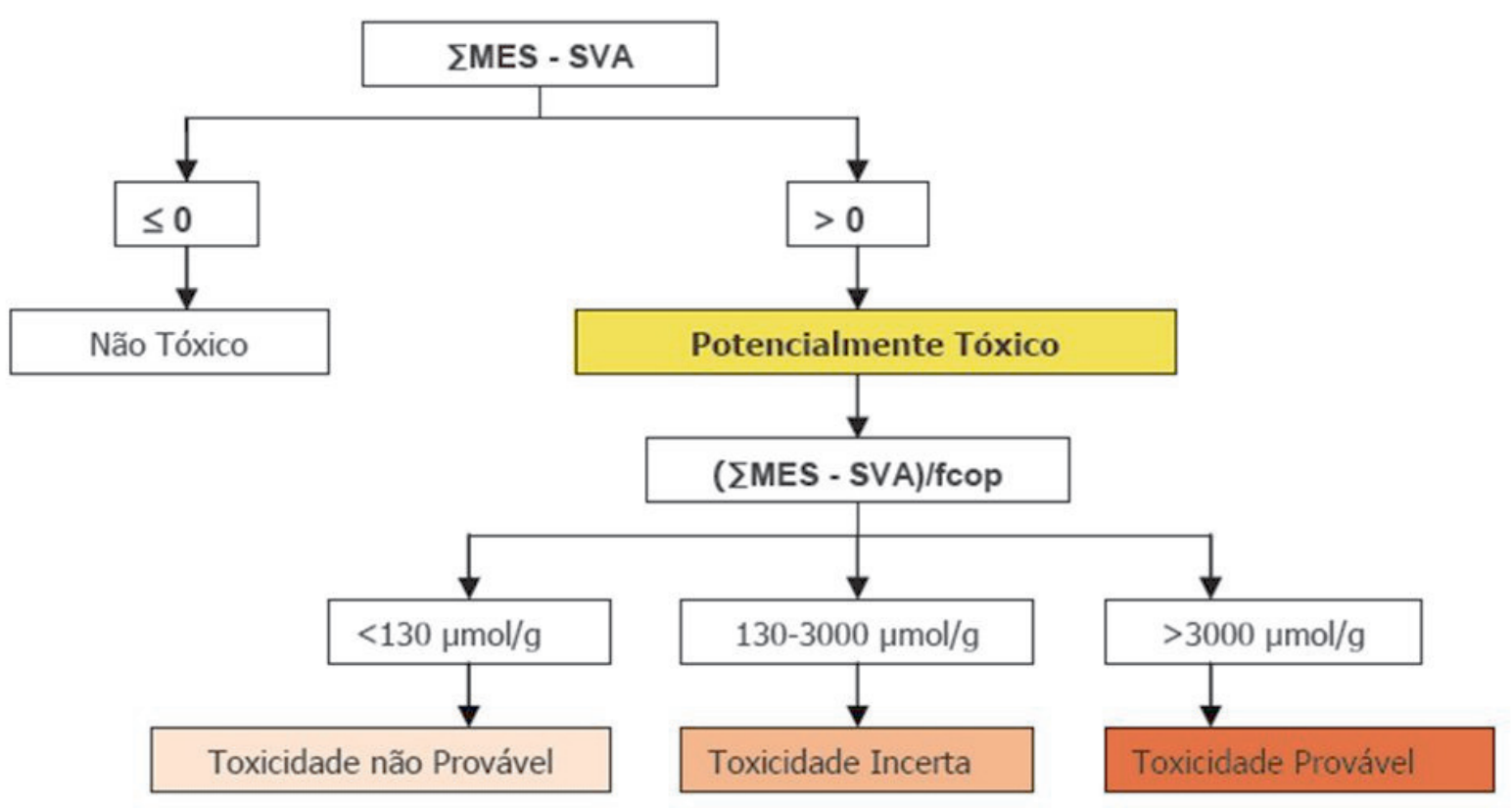

Figura 4 - Fluxograma de interpretação dos valores de MES-SVA e (MES - SVA)/fcop.

como referência para avaliação dos resultados aqui apresentados os valores de SAV/MES obtidos por Silvério (2003) em estudos realizados na represa Rasgão, localizada no Estado de São Paulo e dados do rio Missouri (EUA) que é um rio do porte do rio São Francisco. Além disto, procurou-se realizar uma avaliação coerente com as constatações obtidas em campo das características macroscópicas das amostras de sedimento coletadas.

\section{REFERÊNCIAS}

ANKLEY, G.T, PHIPPS, G.L; LEONARD, E.N., BENDIT, D.A., MATSON, V.R., KOISAN, P.A., COTTER, DIERKES, J.R AND MOHONY, J.D. (1991) - Acid Volatile Sulfide as a factor medianting cadmium and nickel bioavailability in contamined sediments. Environmental toxicol Chemistry. 10:1299 - 1307.

ALLEN, H.E., FU, G. F DENG, B (1993) Analysis of acid volatile sulfide (AVS) and simultaneously extracted metals (SEM) for the estimation of potencial toxicity in aquatic sediments. Environmental toxicological Chemistry. 12:1441-1453. 


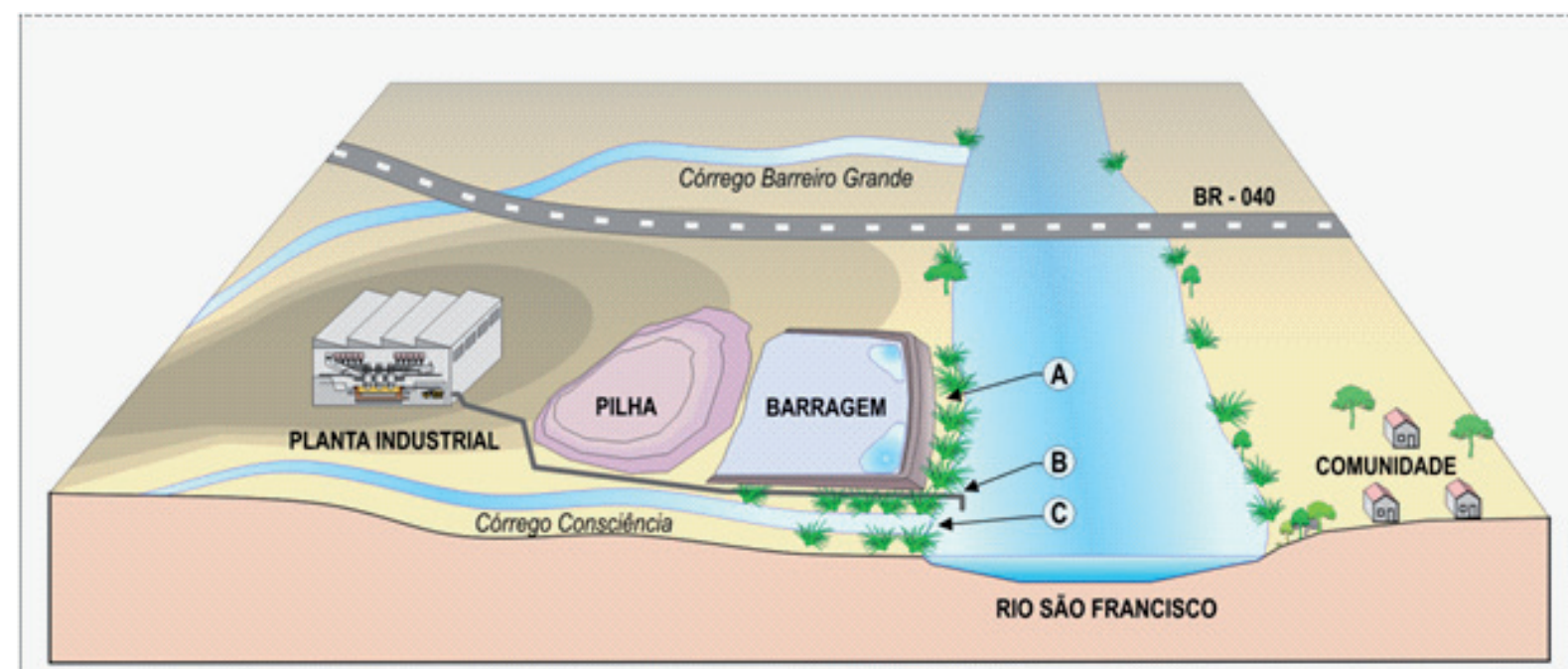

Bloco diagrama da área da planta de zinco da Votorantim Metais.

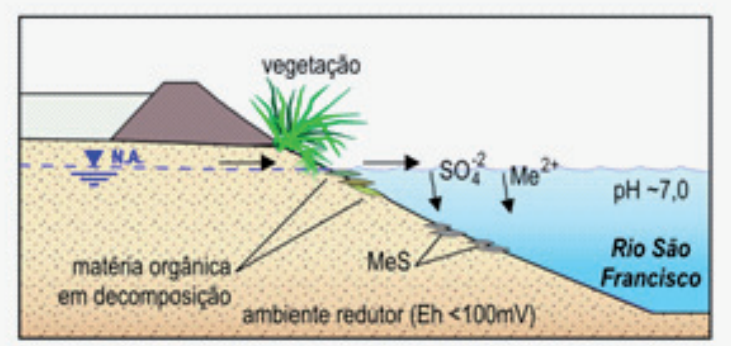

$$
\begin{array}{ll}
\mathrm{pH} & =\text { Accidez da água } \\
\mathrm{Eh} & =\text { Potencial redox da água } \\
\mathrm{Me} & =\text { Metais: } \mathrm{Zn}, \mathrm{Pb}, \mathrm{Cd}, \mathrm{Cu} \\
\mathrm{SO}_{4}^{2} & =\text { Sulfato } \\
\mathrm{MeS} & =\text { Sulfeto metalico precipitado }
\end{array}
$$

Figura A - Encontro da água subterrânea com o rio São Francisco.

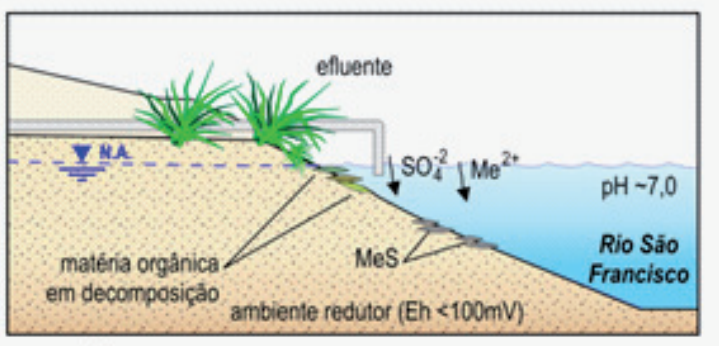

Figura B - Ponto de lançamento de efluente no rio Săo Francisco.

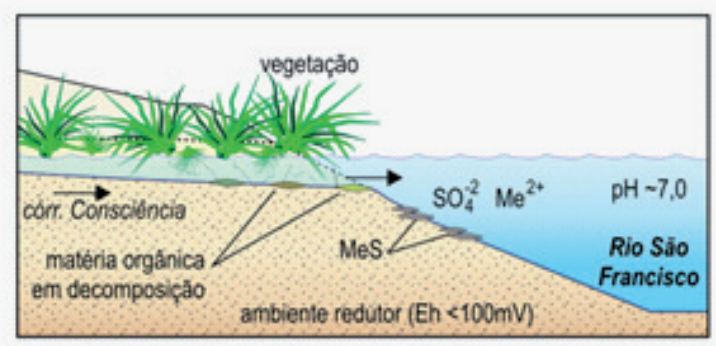

Figura C - Foz do córrego Consciência com o rio Sāo Francisco.

Figura 5 - Representação conceitual do mecanismo geoquímico de precipitação de sulfetos secundários de metais próximos às fontes de contaminação identificadas na planta de zinco da VM-TM.

CONAMA 344. 2004. Estabelece diretrizes gerais e procedimentos mínimos para avaliação do material a ser dragado de áreas brasileiras e dá outras providências. Min. do Meio Ambiente. 25 de março de 2004.

DI TORO, D. M., MAHONEY, J. D., HANSEN, D. J., SCOUT, K. J. HICKS, M. B., MAYR, S. M. E REDMOND, M. S. (1990). Toxicity of Cadmium in Sediments: The role of Acid Volatile Sulphide. Environ. Toxicol. Chem. 9: 1487-1502.
SILVÉRIO, P. F. 2003. Bases técnico-científicas para a derivação de valores-guias de qualidade de sedimentos para metais: experimentos de campo e laboratório. 132 p. Tese (Doutorado em Ciências da Engenharia Ambiental) - Escola de Engenharia de São Carlos, Universidade de São Carlos, São Paulo.

US EPA 1991. Draft Analytical Method for Determination of Acid Volatile Sulfides in Sediment. EPA number: 821R91100. 20 p. 